\title{
Benign Episodic Unilateral Mydriasis: Normal Phenomenon in Migraine? Considerations from A Clinical Case
}

\author{
Alberto Vargas-Cañas ${ }^{1,2^{*}}$ and Catalina Reyes Pérez ${ }^{2}$ \\ ${ }^{1}$ Neurologist, Neurology Unit, Hospital Luis Tisné B, Penalolén, Santiago de Chile, Chile \\ ${ }^{2}$ General Practitioner, Universidad de los Andes de Chile.
}

*Corresponding author: Alberto Vargas-Cañas, Neurologist, Neurology Unit, Hospital Luis Tisné B, Penalolén, Santiago de Chile, Chile.

Published Date: January 08, 2021

\begin{abstract}
In last 50 years, there are many reports about an infrequently condition characterized with a transitory mydriasis associated to migraine attack and without others causes that would explain the ophthalmology finding. This type of headache forces to rule-out critical life-threatening conditions like posterior communicating artery aneurysm among others with neuroimaging test. This conditions has been named Benign Episodic Unilateral Mydriasis (BEUM). We present a clinical case with this characteristics but with the difference that it does not have previous history of migraine like is usual in BEUM. Since there are pupillary alterations in migraine, would the mydriasis be a normal phenomenon in migraine, or a specific isolated disease?
\end{abstract}

Keywords: Red-flags headache; Secondary headache; Migraine; Mydriasis.

\section{Introduction}

Headache accounts for between 1-3\% of admissions to emergency departments (ED), and migraine diagnosis at discharge from ED is variable, ranging from 15-32\% [1]; and the most important issue in ED, is rule-out a secondary headache; and to achieve this goal, a detailed headache anamnesis is mandatory complemented whit a methodical neurological examination in search or the "Red flags" that include sudden onset of headache, onset age after 50 years, increased frequency or severity of headache, new onset of headache in patients with underlying medical condition, or headache with concomitant systemic illness, focal neurologic sings or symptoms, papilledema and post head trauma headache [2]. From all this "Red flags", according some authors, abnormal results from neurological examination are considerate the best clinical parameters to predict a structural intracranial pathology on CT-scan [3].
The concomitance of mydriasis associated to a headache attack meet the criteria to be considered a "Red flag"; and in ED forces to rule-out critical life-threatening conditions with neuroimaging test; but, since the 70s, many patients has been described with a rare condition of mydriasis and ipsilateral head pain without intracranial pathology [4]; and that over the time better characterized, and have some common clinical characteristics: is most frequently observed in female patients, ranking in age from 5 to 53 years, and with typical migraine symptoms [5].

\section{Clinical Case}

Female patient, 29 years old; with no previous morbid or chronic migraine history; who does consult to ED of our hospital, whit a history of 02 hours of holocranial pulsating headache of moderate intensity, associated to nausea, odorphobia, photophobia 
and right blurred vision. The neurological examination show a right mydriasis no reactive to light (Figure 1).
There was no red eye, palpebral retraction or ptosis, and extraocular movements and vision were normal (Figure 2).

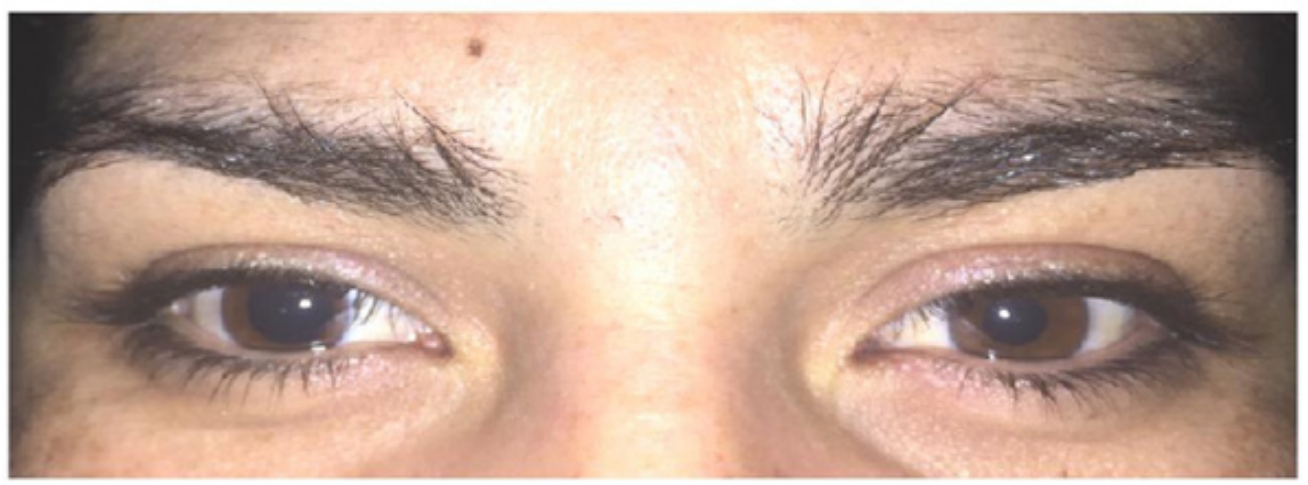

Figure 1: Right mydriasis not reactive to light.
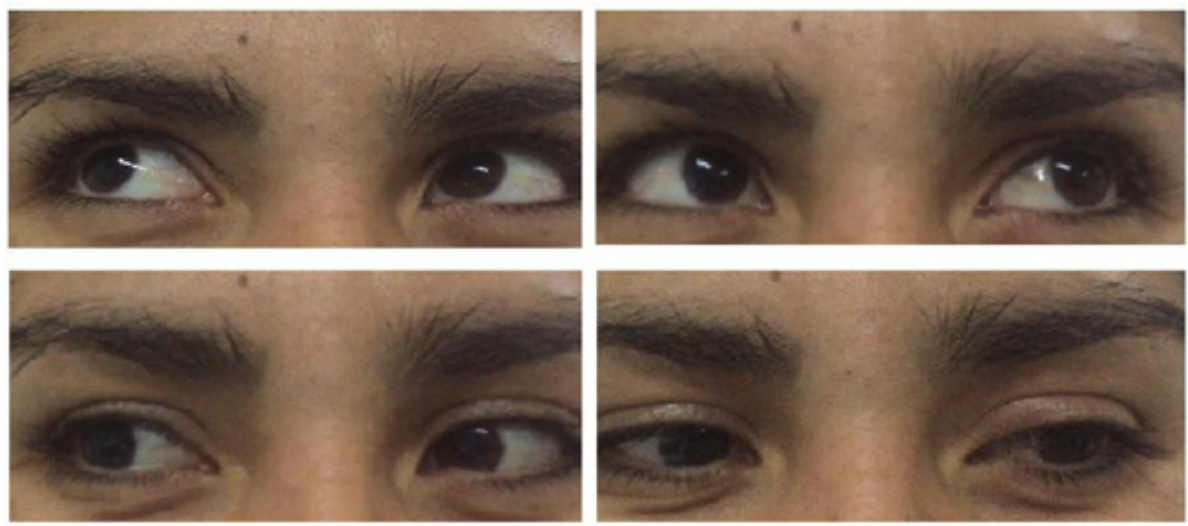

Figure 2: Image showing normal oculomotility.

A CT angiography of the cerebral and neck arteries was made, with negative findings for hemorrhage, intracranial expansive process and aneurysmal dilatations. According local ED protocols, the patient was treated with intravenous steroids and ketoprophen. Symptoms resolve in 02 hours, and the mydriasis in 12 hours. Patient was discharged to home.

\section{Discussion}

BEUM is characterized by pupillary dilation that occurs with transient and intermittent, reversible episodes; associated or not with headache, and without other neurological alarm symptoms or signs [6]. In most cases, it occurs in patients with a personal or family history of migraine [5]; and it is more common in young women [7], although there are cases reported in children [8].

The attacks of BEUM, may be associated with other visual symptoms, like blurred vision, orbital pain, red eye and photophobia; with variable duration of the episode from minutes to days and even more to a week. In most cases, it is unilateral in presentation, but can even alternate in in different episode [9].

Our clinical case, meet many of this criteria like female gender, young age, unilateral ocular findings, blurred vision, nausea, photophobia, and migraine headache findings. Especially attention in our patient was the fact of a migraine debut, and no personal history of migraine.

The pathophysiology of BEUM is not fully understood; the first descriptions assumed that it is a variant of ophthalmoplegic migraine (OM) [4], and until some years ago, this idea was sustained because the $\mathrm{OM}$ has ptosis, diplopia, divergent strabismus and pupillary alteration [10]. This last finding, is explained by intrinsic function alteration of third cranial nerve without comprise of external motility [11]. Other authors, considered the BEUM like a migraine with aura, due is a transient and reversible focal neurological symptom that usually precedes or sometime accompany the headache [10]. But, in contraposition, some reports considered the BEUM a symptom associated with migraine without aura just like photophobia, odorphobia among others [5]. This point of view, is supported by conclusions from literature that suggest pupil size and response in migraine headache suffers have a subtle sympathetic and parasympathetic pupil abnormalities [1214], specifically a parasympathetic hypoactivity of the iris sphincter vs sympathetic hyperactivity in the iris dilator with the consequent mydriasis. Is it possible that our clinical case meet this criterion? 


\section{Conclusion}

Undoubtedly, the mydriasis associated to headache is a "Red flag" that need to ruled-out life-threatening conditions; by this reason, all this patients must to be evaluated with neuroimaging. In cases where secondary causes of headache have been ruled-put a possible diagnosis of Benign Episodic Unilateral Mydriasis would be considered. This condition have some clinical characteristic like be present in migranous suffers, but it can also occur in patients without migraine history.

More studies are need to clarify if BEUM is an isolated condition, or if is part of the migraine spectrum, and to clarify if mydriasis is a normal phenomenon in migraine. By this reason is necessary more reports and try to reach consensus for specific diagnosis criteria.

\section{Acknowledgement}

None.

\section{Conflict of Interest}

No conflict of interest.

\section{References}

1. Cerbo R, Villani V, Gianluca Bruti, Fabrizio Di Stani, Claudio Mostardini (2005) Primary headache in Emergency Department: prevalence, clinical features and therapeutics approach. J Headache Pain 6: 287289.

2. C.Randall Clinch (2001) Evaluation of Acute Headache in Adults. Am Fam Physician 63: 685-692.

3. Ulrich Igor Mbessoh Kegne, Callixte Kuate Tegueu, Dorothée Soh Mankong, Maggy Mbede, Ulrich Gael Tene, et al. (2020) Clinical predictor of significant intracranial computed tomography scan findings in adults experiencing headache disorder. Pan African Medial Journal: 35-81.

4. Hallet M, Cogan D (1970) Episodic Unilateral Mydriasis in Otherwise Normal Patients. Arch Ophtal: 130-136.

5. Woods D, O'Connor P, Fleming R (1984) Episodic Unilateral Mydriasis and Migraine. Am J Ophtal 98: 229-239.

6. Martin Santana I, Gonzalez Hernandez A, L Tandón Cárdenes, P López Méndez, et al. (2015) Benign episodic mydriasis. Experience in a specialist neuro-ophtalmology clinic of a tertiary hospital. Neurologia 30(5): 290-294.

7. Jacobson DM (1995) Benign episodic unilateral mydriasis clinical characteristics. Ophtalmology 102: 1623-1627.

8. Balaguer-Santamaría, C Escofet-Soteras, G Chumbe-Soto, J EscribanoSubias, et al. (2000) Benign episodic unilateral mydriasis. A report of a case in a child. Rev Neurol 31: 743-745.

9. Skeik N, Jabr FI (2011) Migraine with benign episodic unilateral mydriasis. Int J Gen Med 4: 501-513.

10. Headache Classification Subcommittee of the International Headache Society. The International Classification of Headache Disorders: 3rd edition (ICHD-III). Available in http://ihs-classification.org/en/ HersheyAD.

11. BekS, Genc G, Demirkaya S, Eroglu E, Odabasi Z (2009) Ophthalmoplegic migraine. The Neurologist 15: 147-149.

12. Harle E D, Wolffshon J, Bruce J W Evans (2005) The pupillary light reflex in migraine. Ophtal Physiol Opt 25: 240-245.

13. Cortez M, Rea Natalie, et al. (2017) Altered pupillary light response scales with disease severity in migrainous photophobia. Cephalalgia.

14. Cambron M, Maertens H, Koen Paemeleire, Luc Crevits (2014) Autonomic Function in Migraine Patients: Ictal an Interictal Pupillometry. Headache 54: 655-662. 